\title{
Limited Diffraction Maps for Pulsed Wave Annular Arrays
}

\author{
Fox, Paul D.
}

Published in:

Proceedings. IEEE Ultrasonics Symposium, 2002.

Publication date:

2002

Document Version

Publisher's PDF, also known as Version of record

Link back to DTU Orbit

Citation (APA):

Fox, P. D. (2002). Limited Diffraction Maps for Pulsed Wave Annular Arrays. In Proceedings. IEEE Ultrasonics Symposium, 2002. (pp. 509-512). IEEE.

\section{General rights}

Copyright and moral rights for the publications made accessible in the public portal are retained by the authors and/or other copyright owners and it is a condition of accessing publications that users recognise and abide by the legal requirements associated with these rights.

- Users may download and print one copy of any publication from the public portal for the purpose of private study or research.

- You may not further distribute the material or use it for any profit-making activity or commercial gain

- You may freely distribute the URL identifying the publication in the public portal

If you believe that this document breaches copyright please contact us providing details, and we will remove access to the work immediately and investigate your claim. 


\title{
Limited Diffraction Maps for Pulsed Wave Annular Arrays
}

\author{
Paul D Fox ${ }^{*}$
}

\begin{abstract}
A procedure is provided for decomposing the linear field of flat pulsed wave annular arrays into an equivalent set of known limited diffraction Bessel beams. Each Bessel beam propagates with known characteristics, enabling good insight into the propagation of annular fields to be obtained. Numerical examples are given in the context of a 10-ring annular array operating at a central frequency of $2.5 \mathrm{MHz}$ in water.
\end{abstract}

\section{INTRODUCTION}

This article describes a method for decomposing the field of flat pulsed wave (PW) annular arrays into a set of known $J_{0}$ Bessel beam $[1,2]$ subfields by using a $1 D$ Fourier-Bessel series. In [3] the continuous wave (CW) case was considered, and this paper extends it to the PW case. The technique generates a straightforward numerical tool for obtaining and insight into the propagation of annular PW fields. See [4, 3] for more detailed background information on the technique and $[5,6,7,3,8]$ for information on Bessel beams.

\section{PW ANNUlaR ARRAYS}

Consider a fiat annular $N$-ring PW array of radius $R$ with surface pressure $q(r, t)$, spatially quantised in the radial $r$ direction due to the ring structure. This leads to $N$ stepwise-constant ring pressures $q_{p}(t)$, where $p=$ $1, \ldots, N$ is the ring number and $p=1$ for the inner ring with $p=N$ for the outer ring. Assume also a sampled pulsed system, enabling representation by the discrete Fourier sum

$$
\begin{gathered}
q_{p}\left(t_{d}\right)=\sum_{s=0}^{n_{\omega}} Q_{p}\left(\omega_{s}\right) e^{-j \omega_{s} t_{d}} \\
\omega_{s}=s \cdot 2 \pi f_{0}
\end{gathered}
$$

${ }^{*}$ Center for Fast Ultrasound Imaging, Ørsted-DTU, Building 348, Technical University of Denmark, DK-2800 Kgs. Lyngby, Denmark. (pdf@oersted.dtu.dk) where $n_{\omega}$ is the number of nonzero Fourier frequencies, $f_{0}$ denotes fundamental frequency in $\mathrm{Hz}$, and $t_{d}$ a discrete sampling point in time. Then $Q_{p}\left(\omega_{s}\right)$ may be obtained in practice by taking the Fourier transform $Q_{p}\left(\omega_{s}\right)=F F T\left\{q_{p}\left(t_{d}\right)\right\}$ of the surface quantisation profile, and the entire array of all $N$ rings may then be represented as

$$
\left[\begin{array}{c}
q_{1}\left(t_{d}\right) \\
\vdots \\
q_{N}\left(t_{d}\right)
\end{array}\right]=\sum_{s=0}^{n_{\omega}}\left[\begin{array}{c}
Q_{1}\left(\omega_{s}\right) \\
\vdots \\
Q_{N}\left(\omega_{s}\right)
\end{array}\right] e^{-j \omega_{s} t_{d}}
$$

\section{LIMITED DIFFRACTION BESSEL BEAMS}

Annular arrays have circular symmetry propagation which, in the lossless linear case, is dictated by the wave equation

$$
\left[\frac{1}{r} \frac{\partial}{\partial r}\left(r \frac{\partial}{\partial r}\right)+\frac{\partial^{2}}{\partial z^{2}}-\frac{1}{c^{2}} \frac{\partial^{2}}{\partial t^{2}}\right] f(r, z, t)=0
$$

where $f(r, z, t)$ is the scalar field value, $r$ is the radial distance from the cylindrical centerline, and $z$ is the outward propagation distance perpendicular to the transducer surface. The transducer sits in the $z=0$ plane, centered around $r=0$, and in a medium with speed of sound $c$ (assumed real). Then equation (3) has an infinite number of Bessel beam solutions [2] of the form

$$
\begin{aligned}
& f_{i}\left(r, z, t, \omega_{s}\right)=J_{0}\left(\alpha_{i} r\right) \cdot e^{j \beta_{i}\left(\omega_{s}\right) z} \cdot e^{-j \omega_{s} t} \\
& \beta_{i}\left(\omega_{s}\right)=\sqrt{k_{s}^{2}-\alpha_{i}^{2}}: k_{s}=\omega_{s} / c
\end{aligned}
$$

where $k_{s}=\omega_{s} / c$ is the wavenumber (assumed real), and $\alpha_{i} \geq 0$ and $\beta_{i}\left(\omega_{s}\right)$ are the propagation parameters in the radial and axial directions $r, z$ respectively. Notice that for $\beta_{i}\left(\omega_{s}\right)>\alpha_{i}$ the wave becomes evanescent in the $z$ direction, whereas for all $\beta_{i}\left(\omega_{s}\right) \leq \alpha_{i}$ the wave propagates nonevanescently. In this latter case, the beams have been shown to propagate similarly to those 
of an axicon transducer $[1,2]$ with axicon angle $\zeta_{i}\left(\omega_{s}\right)$ and depth of field $D O F_{i}\left(\omega_{s}\right)$ given by

$$
\begin{aligned}
& \operatorname{DOF}_{i}\left(\omega_{s}\right) \approx R \sqrt{\left(\frac{k_{s}}{\alpha_{i}}\right)^{2}-1} \\
& \zeta_{i}\left(\omega_{s}\right)=\sin ^{-1}\left(\frac{\alpha_{i}}{k_{s}}\right)
\end{aligned}
$$

The key idea in this paper is therefore to model the quantised surface pressure of the transducer by a weighted set of Bessel functions with different $\alpha_{i}$ parameters, since each Bessel function then corresponds to a limited diffraction Bessel beam with known propagation characteristics. The immediate question however is how to chose the appropriate $\alpha_{i}$ parameters and how to establish what the relative weightings of the different subfields in the system should be. The solution comes from the application of Fourier-Bessel series.

\section{Use of Fourier-Bessel SERIES}

A 1D Fourier-Bessel series may be used to model the quantised surface pressure $Q\left(r, \omega_{s}\right)$ over the range $0 \leq$ $r \leq R$ at each frequency $\omega_{s}$ by an infinite set of known basis Bessel functions :

$$
\begin{aligned}
& Q\left(r, \omega_{s}\right)=\sum_{i=1}^{\infty} A_{i}\left(\omega_{s}\right) \cdot J_{0}\left(\alpha_{i} r\right) \\
& \alpha_{i}=x_{i} / R \quad: \quad J_{0}\left(x_{i}\right)=0 \\
& A_{i}\left(\omega_{s}\right)=\frac{2}{R^{2} J_{1}^{2}\left(x_{i}\right)} \int_{0}^{R} Q\left(r, \omega_{s}\right) \cdot r J_{0}\left(\alpha_{i} r\right) \cdot d r
\end{aligned}
$$

where $J_{0}(\cdot)$ is the Bessel function of the first kind of order zero and $x_{i}$ in (6) are the known infinite set of real monotonically increasing positive solutions to $J_{0}\left(x_{i}\right)=$ 0 . The annular spatial profile $Q\left(r, \omega_{s}\right)$ for a given $\omega_{s}$ is stepwise constant $Q\left(r, \omega_{s}\right)=Q_{p}\left(\omega_{s}\right)$ for the $N$ rings $p=1, \ldots, N$ and this allows the integral for $A_{i}\left(\omega_{s}\right)$ in (6) to be evaluated analytically as

$$
\begin{aligned}
& A_{i}\left(\omega_{s}\right)=\sum_{p=1}^{N} C_{i, p} \cdot Q_{p}\left(\omega_{s}\right) \\
& C_{i, p}=\frac{2\left[r_{p}^{+} J_{1}\left(\alpha_{i} r_{p}^{+}\right)-r_{p}^{-} J_{1}\left(\alpha_{i} r_{p}^{-}\right)\right]}{R x_{i} J_{1}^{2}\left(x_{i}\right)}
\end{aligned}
$$

where $J_{1}(\cdot)$ is the first-order Bessel function of the first kind and $r_{p}^{-}, r_{p}^{+}$are inner and outer radii of ring $p$

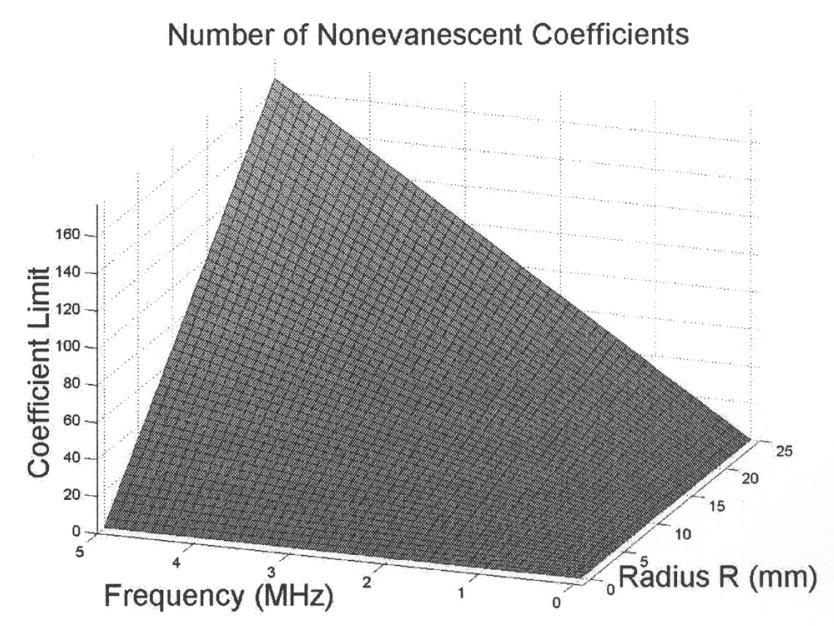

Figure 1: Number of nonevanescent coefficients for $c=1500 \mathrm{~m} / \mathrm{s}$ as a function of frequency and transducer radius

respectively. Note here that the quantities $A_{i}\left(\omega_{s}\right)$ are generally complex since $Q_{p}\left(\omega_{s}\right)$ are generally complex $\left(C_{i, p}\right.$ are real). Furthermore, since $\alpha_{i}=x_{i} / R$ increases monotonically a value of index $i$ is always reached at which $\alpha_{i}>k_{s}$ and the corresponding beam becomes evanescent since then $\beta_{i}\left(\omega_{s}\right)$ becomes imaginary. This limit $i=l\left(k_{s}, R\right)$ may be obtained analytically by replacing $k$ with $k_{s}$ in the expression for $l(k, R)$ derived previously in $[4,8]$ to obtain

$$
l\left(k_{s}, R\right) \approx \frac{k_{s} R}{\pi}+\frac{1}{4}
$$

which is proportional to wavenumber $k_{s}$ and modelling aperture $R$, but independent of any particular transducer pressure $Q\left(r, \omega_{s}\right)$ (see Figure 1 for typical numerical values). As a result, the infinite sum in (4) becomes replaced by a finite sum of known nonevanescent limited diffraction beams :

$$
f\left(r, z, t, \omega_{s}\right)=\sum_{i=1}^{l\left(k_{s}, R\right)} A_{i}\left(\omega_{s}\right) \cdot J_{0}\left(\alpha_{i} r\right) \cdot e^{j \beta_{i}\left(\omega_{s}\right) z} \cdot e^{-j \omega_{s} t}
$$

\section{Summary of Analysis TeChnique}

The full technique for the field analysis then comprises four simple steps : 


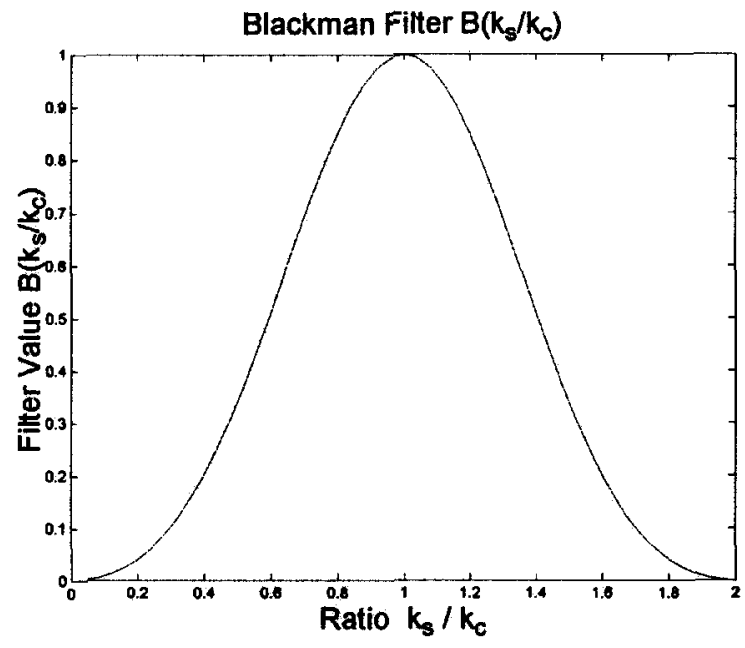

Figure 2: Blackman window

- Model the array as a set of Fourier sums (Eqn 1,2)

- Obtain coefficients $\alpha_{i}$ from Bessel roots (6) and find maximum index $i=l\left(k_{s}, R\right)$ after which evanescence begins (i.e. $\alpha_{i}>k_{s}$ )

- Apply Fourier-Bessel series (6) to obtain all nonevanescent subfield weighting coefficients $A_{i}\left(\omega_{s}\right)$ from (7)

- Find which are the main subfield components $A_{i}\left(\omega_{s}\right)$ and examine their corresponding propagation characteristics are from Eqn (5)

\section{NUMERICAL EXAMPLES}

Take the $J_{0}$ Bessel transducer of $\mathrm{Lu}$ and Greenleaf described in [5]. This is an $N=10$-ring with outer radius $R=25 \mathrm{~mm}$ operating in water $(c=1500 \mathrm{~m} / \mathrm{s})$ at a center frequency of $2.5 \mathrm{Mhz}$ (central wavenumber $k_{c}=10,471.98 \mathrm{~m}^{-1}$ ), and with Blackman window transfer function

$$
B\left(k_{s}\right)=0.42-0.5 \cos \left(\frac{\pi k_{s}}{k_{c}}\right)+0.08 \cos \left(\frac{2 \pi k_{s}}{k_{c}}\right)
$$

Now consider three different examples of transducer surface pressure profiles. The first is a zero-order $\mathrm{X}$ wave, which by definition has frequency domain ring pressures

$$
Q_{p}\left(\omega_{s}\right)=B\left(\omega_{s}\right) \cdot e^{-a_{0} k_{s}} \cdot J_{0}\left(k_{s} r_{p} \sin \zeta\right)
$$

in which $a_{0}=0.05 \mathrm{~mm}, \zeta=4^{\circ},(\sin \zeta=0.07)$. Figure 3 shows nonevanescent coefficients for an exact $X$ wave (left) and the quantised $X$ wave (right). Notice that extra subfields are introduced in the quantised $\mathrm{X}$ wave, reflecting the fact that the exact $X$ wave cannot be implemented due to the constraints of the stepwise annular structure. The second example is a focused Gaussian beam with frequency domain representation

$$
Q_{p}\left(\omega_{s}\right)=B\left(\omega_{s}\right) \cdot e^{-\tau_{p}^{2} / \sigma^{2}} \cdot e^{j k_{s}\left(F-\sqrt{F^{2}+r_{p}^{2}}\right)}
$$

Figure 4 shows the beam for fixed $\sigma=5 \mathrm{~mm}$ and two different focal distances $F=60 \mathrm{~mm}$ (left) and $F=120 \mathrm{~mm}$ (right). Figure 5 (left) then also shows the focused Gaussian for a different $\sigma=5 \mathrm{~mm}$ at focus $F=120 \mathrm{~mm}$. Notice from these that a change in $e i-$ ther the Gaussian phase term $F$ or the magnitude term $\sigma$ affect both the phase and magnitude of the weighting coefficients $A_{i}\left(\omega_{s}\right)$. Finally, also take a plane wave (Figure 5, right) for which

$$
Q_{p}\left(\omega_{s}\right)=B\left(\omega_{s}\right)
$$

A 'pure' plane wave corresponds to an $\alpha$ parameter of $\alpha=0$, since $J_{0}(0)=0$ by definition. However, in the piston implementation we see nonzero weighting coefficients appearing for the Fourier-Bessel parameters $\alpha_{i}>0$ and these correspond to subfields generating the edge waves which are observed in practice from a piston transducer. (See [4] and [3] for helpful insights).

\section{CONCLUSIONS}

A method has been given for finding the linear emitted field of flat annular arrays in terms of a set of known limited diffraction subfields. The technique is numerically straightforward and gives a helpful insight into the propagation of annular fields. Extension to non-annular arrays is possible by including higher order Bessel terms.

\section{ACKNOWLEDGEMENT}

This work was partially sponsored by grant 26-01-0178 from the Danish Science Foundation, Denmark. 

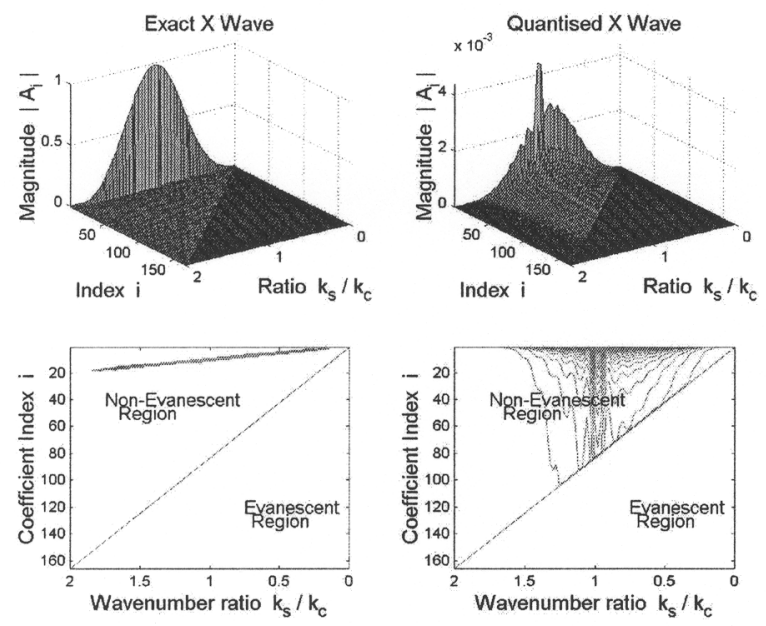

Figure 3: Exact $\mathrm{X}$ wave (left upper) and quantised $\mathrm{X}$ wave (right upper). Also contour plots (lower) of nonevanescent coefficients.
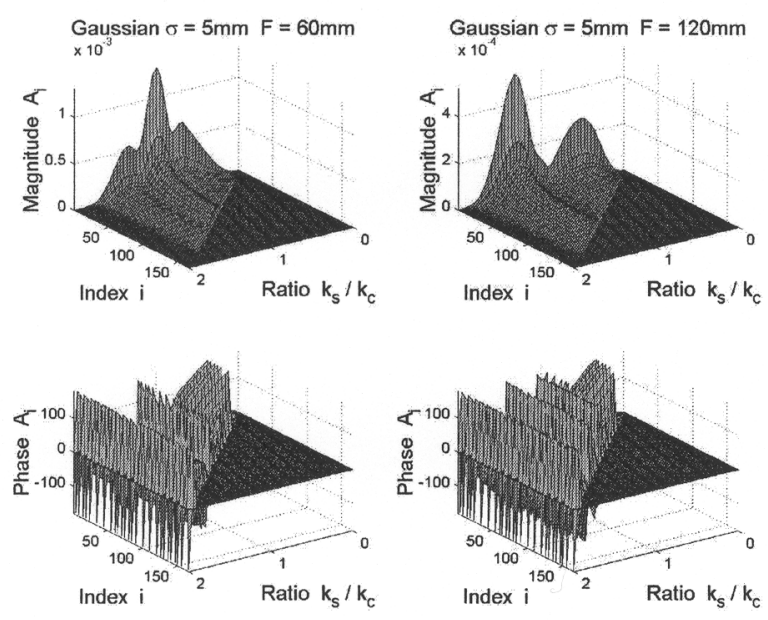

Figure 4: Focused Gaussian for different focal parameters $F=60 \mathrm{~mm}$ (left) and $F=120 \mathrm{~mm}$ (right). Notice changes in both phase and amplitude of weighting coefficients.
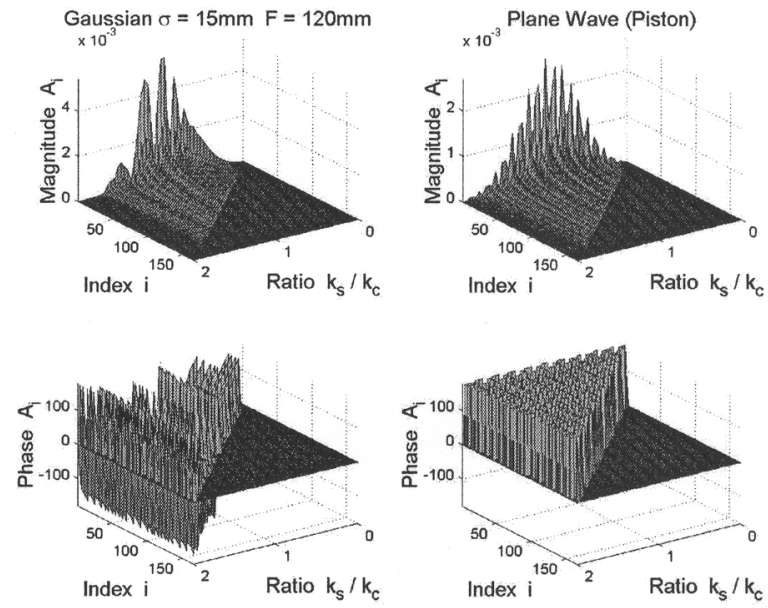

Figure 5: Focused Gaussian for different $\sigma=15 \mathrm{~mm}$ (left) and piston plane wave (right).

\section{REFERENCES}

[1] J. A. Stratton, Electromagnetic Theory, p. 356, McGrawHill Book Company, New York and London, 1941.

[2] J. Durnin, "Exact solutions for nondiffracting beams. I. The scalar theory," J. Opt. Soc. Am. A, vol. 4, no. 4, pp. 651-654, 1987.

[3] P. D. Fox and S. Holm, "Modelling of CW annular arrays using limited diffraction Bessel beams," IEEE Trans. Ultrason., Ferroelect., Freq. Contr., vol. 49, no. 1, pp. 8593, 2002.

[4] P. D. Fox and S. Holm, "Decomposition of acoustic fields in quantised Bessel beams," Ultrasonics, vol. 38, pp. 190-194, March 2000.

[5] J.-Y. Lu and J. F. Greenleaf, "Ultrasonic nondiffracting transducer for medical imaging," IEEE Trans. Ultrason., Ferroelect., Freq. Contr., vol. 37, no. 5, pp. 438-447, September 1990.

[6] S. Holm, "Bessel and conical beams and approximation with annular arrays," IEEE Trans. Ultrason., Ferroelect., Freq. Contr., vol. 45, no. 3, pp. 712-718, May 1998.

[7] P. R. Stepanishen, "A generalized modal impulse response and Fourier transform approach to investigate acoustic transient Bessel beams and Bessel bullets," J. Acoust. Soc. Am., vol. 105, no. 3, pp. 1493-1502, March 1999.

[8] P. D. Fox, J. Cheng, and J.-Y. Lu, "Fourier-bessel field calculation and tuning of a CW annular array," IEEE Trans. Ultrason., Ferroelect., Freq. Contr., vol. To appear, 2002. 